\title{
Fatigue life and fracture mechanics of unconstrained Ni-Mn-Ga single crystals in a rotating magnetic field
}

\author{
T. Lawrence ${ }^{1}$, P. Lindquist ${ }^{1}$, K. Ullakko ${ }^{2}$, P. Müllner ${ }^{1 \#}$ \\ ${ }^{1}$ Department of Materials Science and Engineering, Boise State University, Boise, ID 83725, \\ USA \\ ${ }^{2}$ Lappeenranta University of Technology, LUT Chemtech, Savonlinna, Finland \\ \#Corresponding author email: petermullner@boisestate.edu
}

\begin{abstract}
Fracture resistance and long fatigue life are required to commercialize Ni-Mn-Ga ferromagnetic shape memory alloys. While Ni-Mn-Ga achieves high fatigue life at low strains, there is little data on the fatigue life of samples that are actuated near the theoretical strain limit. Furthermore, constraints imposed by clamping samples impact their magneto-mechanical and fatigue properties. Here, $10 \mathrm{M}$ Ni-Mn-Ga single crystals were exposed to a rotating magnetic field while holding them with rubber O-rings so they were minimally constrained. Prior to testing, sample surfaces were prepared to various levels of finish. Fatigue life varied with some samples reaching more than 800,000 cyclic loads before fracturing and some samples failing after only 4,000 cyclic loads. Long fatigue life and fracture resistance are achieved when avoiding crystal defects and surface imperfections. Short fatigue life was caused by high surface roughness and stress concentration at defects and notches.
\end{abstract}

\section{Introduction}

Ferromagnetic shape memory alloys (FSMAs) such as Ni-Mn-Ga exhibit large reversible strains in a variable magnetic field [1], where twin boundary motion accommodates magneticfield-induced strain (MFIS) [2,3]. Unlike conventional shape memory alloys, which require heating and cooling for use as actuators, shape change of FSMAs is much faster when a magnetic field is used. Magnetic-field-induced actuation of FSMAs results in strains as high as $12 \%$ for Ni-Mn-Ga alloys [4] with frequencies as high as $2 \mathrm{kHz}$ [5]. Recent results show that twin boundaries accelerate to speeds of several meters per second in a few microseconds [6] which suggests actuation frequencies of hundreds of $\mathrm{kHz}$.

The shape memory properties of Ni-Mn-Ga make it an ideal candidate for applications including micro-fluid pumps, energy harvesting, and memory devices [7-11]. For these uses, long fatigue life and fracture resistance are required when FSMAs are actuated thousands or even millions of times and failure caused by nucleation and propagation of cracks must be prevented. 
Mechanical and mangeo-mechanical properties of Ni-Mn-Ga has been studied extensively for nearly twenty years; however, there are few studies on how fatigue affects mechanical properties. Müllner et al. performed fatigue experiments on single crystals with 10M and $14 \mathrm{M}$ martensite indicating that thermomechanical treatments aid in increasing strain during magnetomechanical cycling $[12,13]$. Aaltio et al. mechanically actuated crystals with 10M martensite in tension and compression to $2 \times 10^{9}$ cyclic loads [13, 14]. These samples fractured along macroscopic $\{111\}$ planes in $<110]$ directions. Effectively trained samples exhibit the highest strains and shortest fatigue life [15]. Straka et al. [16] demonstrated enhanced fatigue life for samples with tailored fine twin microstructures as suggested in an earlier study[17]. Constraining the FSMA element inhibits twin reorientation locally within the constrained volume. Surface defects and residual stresses limit twin boundary motion and increases twinning stress [18]. Constrained samples exhibit reduced strain and prolonged fatigue life [19]. To separate material properties from effects of clamping, it is important to study the samples in a variable magnetic field without constraints.

Rotary fatigue experiments were performed on notched and un-notched single crystal samples. Constraints were minimized by using rubber to hold the samples in the testing apparatus. The switching field was measured intermittently after 20,000 actuation cycles to determine the effect of fatigue damage on the effective twinning stress. Samples were prepared to various surface finishes to evaluate the effects of surface roughness on fatigue and fracture.

\section{Experimental procedures}

Six 10M single crystals were grown with a nominal composition of $\mathrm{Ni}_{51.2} \mathrm{Mn}_{26.6} \mathrm{Ga}_{22.2}$ using the Bridgman-Stockbarger technique. The crystals were grown from a seed along the $<100>$ direction and had a diameter of $6 \mathrm{~mm}$. Details of crystal growth parameters are given by Kellis, et al. [20]. X-Ray Diffraction was performed along the length of each crystal using a Bruker D8 to determine the crystal structure, orientation, and lattice parameters. A Hitachi SEM with an Oxford Energy Dispersive X-Ray Spectroscopy detector was used to determine the composition along the length of the crystals.

The $10 \mathrm{M}$ portion of one crystal was cut with a Princeton Scientific precision wire saw into five parallelepipeds with faces parallel to the $\{100\}$ planes (austenite) for compression testing to determine twinning stress. The samples had dimensions of approximately $2 \times 3 \times 6 \mathrm{~mm}$. Samples were loaded into a compression testing instrument (Zwick, Ulm, model 1445) equipped with a $500 \mathrm{~N}$ load cell so that the long axis of the crystal was parallel to the axis of compression. The samples were initially loaded to $0.05 \mathrm{MPa}$ at a crosshead speed of $8.3 \times 10^{-6} \mathrm{~m} / \mathrm{s}$ to preload the sample. Mechanical compression testing was performed at a constant crosshead speed of $2 \times 10^{-6} \mathrm{~m} / \mathrm{s}$ until a maximum stress of $5 \mathrm{MPa}$ was achieved. The samples were then unloaded at a constant crosshead speed of $4 \times 10^{-6} \mathrm{~m} / \mathrm{s}$ until a minimum stress of $0.05 \mathrm{MPa}$ was reached. The resolution of the instrument was better than $0.5 \mathrm{~N}$ in force and $10 \mathrm{~nm}$ in displacement. Three compression tests were performed on each sample. In between compression testing, a magnetic 
field was applied parallel to the short axis to elongate the sample along the long axis for the next test.

Thirty four cylindrical disks each with a thickness of approximately $1.5 \mathrm{~mm}$ and a $6 \mathrm{~mm}$ diameter were cut from the remaining five crystals. The samples were ground flat and polished on both sides by using a mechanical polishing wheel with progressive polishing steps from 1200 to 4000 grit $\mathrm{SiC}$ paper, then finished with $3 \mu \mathrm{m}$ diamond slurry. From these samples, eight were polished further with $1 \mu \mathrm{m}$ diamond slurry, and of these five were additionally polished with a final $0.04 \mu \mathrm{m}$ diamond slurry. To six samples mechanically polished with $3 \mu \mathrm{m}$ diamond slurry, notches were cut through the thickness of the disk with a length $1 \pm 0.2 \mathrm{~mm}$ in the $[100]_{\mathrm{S}}$ and $[110]_{S}$ directions with a Princeton Scientific precision wire saw with $50 \mu \mathrm{m}$ wire thickness. The surface roughness of the samples was measured using a Veeco Wyko optical profilometer.

A MicroSense Model 10 vibrating sample magnetometer (VSM) was used to measure magnetic properties and the switching field for all samples (the field at which twin boundaries move) [21]. The designation [uvw] $]_{S}$ and $(\mathrm{hkl})_{S}$ will be used to identify directions and planes related to the sample coordinate system and crystallographic orientation. Samples were initially loaded so that the magnetic field was parallel to the $[010]_{S}$ direction and parallel to the sample face. The sample was fully magnetized in this orientation by applying an increasing magnetic field up to $1.6 \mathrm{MA} / \mathrm{m}$. The magnetic field was then turned off and the sample was rotated by $90^{\circ}$ so that the pole pieces were perpendicular to the sample face and parallel to the $[001]_{S}$ direction. An increasing magnetic field up to $0.8 \mathrm{MA} / \mathrm{m}$ was applied to measure the switching field in this orientation. The field was zeroed again, the sample was then rotated back to its original position so that the magnetic field was parallel to $[010]_{S}$ and the switching field was measured in this orientation. This process was repeated until the switching field was measured three times in both the $[010]_{S}$ and $[001]_{S}$ directions.

Fatigue testing was performed in a custom made apparatus using a modified lathe. The samples were placed in a sample holder capable of accommodating 60 samples on the tailstock. The samples were oriented such that the $[100]_{S}$ direction was parallel to the axis of rotation of the spindle and the $[001]_{S}$ direction was perpendicular to the axis of rotation. The spindle was replaced by a sleeve containing a central diametrically magnetized cylindrical permanent magnet and two rectangular permanent magnets (Figure 1). The sample holder was slid into the sleeve so that the samples were in between the central magnet and the outer magnets. As the spindle rotated the samples were exposed to a full reversal of the magnetic field for each revolution of the spindle assembly. The maximum magnetic field perpendicular and parallel to the sample face was $0.52 \mathrm{MA} / \mathrm{m}$ and $0.41 \mathrm{MA} / \mathrm{m}$, respectively. The radial and tangential fields at the sample position as a function of rotation angle are shown in Figure 1b. Samples were inspected in a Leica microscope using differential interference contrast (DIC) after 10,000 revolutions (i.e. 20,000 magneto-mechanical cycles [12]) to monitor crack initiation and propagation.

\section{Results}


The structure and composition along the axis of the crystal obtained with XRD and EDS scans are shown in Figure 2. The manganese concentration increased, while the nickel and gallium concentration decreased along the growth direction. The crystal structure starting at the seed end of the crystal, was $10 \mathrm{M}$ for the first $17 \mathrm{~mm}$ which transistioned to a mixture of $10 \mathrm{M}$ and $14 \mathrm{M}$ structure, then a segment with $14 \mathrm{M}$, followed by a final segment of non-modulated martensite. Samples used in fatigue and compression testing were cut from the $10 \mathrm{M}$ portion of the crystals with composition ranges given in Table 1.

Magnetization curves measured before and after cycling a sample 810,000 times are shown in Figure 3. The differences between the magnetization curves measured with the magnetic field parallel and perpendicular to the sample face is due to the differences in the demagnetizing fields in these directions. The negative slope of the magnetization curve is due to a large increase in the demagnetization field of the sample as the structure reorients and the moments align parallel to the magnetic field with very little change in applied magnetic field. After extended cyclical loading the discontinuity is less pronounced indicating a reduction of twinning activity.

The switching field was measured to determine how the twinning stress varied over the fatigue life. The effective twinning stress was determined from models by Chernenko and L'Vov with consideration of the demagnetizing field $[22,23]$. While the applied magnetic field was increased monotonically, the effective field in the sample decreases when twin boundaries move rapidly. This leads to a zig-zag shape of the magnetization vs. effective field curve (e.g. Fig. 3b). The effective twinning stress was evaluated from the effective magnetic field which triggered twin boundary motion. The average effective twinning stresses measured in switching field tests with the magnetic field applied parallel to the $[010]_{S}$ and $[001]_{S}$ directions from samples prepared with similar methods are listed in Table 2. The range in effective twinning stress calculated for the fatigue samples from Crystals $1-5$ was $0.20 \pm 0.07$ to $1.0 \pm 0.3 \mathrm{MPa}$, which was comparable to the average twinning stress measured in compression tested samples from Crystal 6 ranging from $0.3 \pm 0.2$ to $0.8 \pm 0.1 \mathrm{MPa}$.

Rotary fatigue samples exhibited three types of characteristic cracks. Type I cracks tended to propagate straight in the $\langle 110\rangle_{\text {S }}$ direction as shown with arrow 1 in Figure 4 . The macroscopic fracture planes for these types of cracks were close to $\{111\}$ and on the fracture surface long striations similar to river patterns typical for brittle fracture [24] were observed, Figure 5. The striations pointed towards crack initiation points. Type II cracks (marked with arrow 2 in Figure 4) tended to propagate macroscopically along <100]. On a smaller scale these cracks propagated in <110] directions zigzagging back and forth horizontally across the sample face. And type III cracks (marked with arrow 3 in Figure 4) occurred in these crystals along small angle grain boundaries. Three of the five crystals tested exhibited small angle grain boundaries (SAGBs) near the seed end. 
Notches were cut along the [100] and [110] directions of samples to determine if there is a preferential type of crack that occurs from cyclical loading. Notches induced stress concentrations at the tip of the notch causing cracks to nucleate at low cycle numbers and in a controlled location. Cracks preferentially propagated from the [100] notches in $\langle 110\rangle$ directions (Figure 6). While cracks did propagate from the [110] notches the cracks propagated from the [100] notches in fewer cycles for seven out of the eleven total samples. The fracture plane decoupled portions of the crystals that showed different twin activities as shown in Figure 6 were compound [010] twins and [100] type I and type II twins are only present on the left side of the upper crack and not on its right side. Cracks at the notch tip occurred in as few as 4,000 magneto-mechanical cycles.

The microstructure of twins near the cracks was more complex compared to areas away from the cracks. Away from the cracks there were large horizontal type II twins and near the cracks the type II twins decomposed into a mixture of finely divided type I and II twins, Figure7. Traces of type I twin boundaries are parallel to the [100] direction while traces of type II twins deviate from [100] by approximately $6^{0}[25]$. The twin structure near the cracks was refined with thinner twins while the central area in between cracks contained thicker type II twins. Compound twins running vertically in the [010] direction were only present in the portion where coarse twins were observed. For twin variants containing visible compound twins the $c$-axis of the unit cell was oriented parallel to the face of the sample (i.e. in the [010] direction). For variants where the compound twins were not visible, the $c$-axis was perpendicular to the sample face (i.e. in the [001] direction). The compound twins indicate which domain contains the different modulation directions of the unit cell.

To compare fatigue life, samples were split into three groups depending on average surface roughness, $R_{\mathrm{a}}$. These groups are $R_{\mathrm{a}}<30 \mathrm{~nm}, 30 \mathrm{~nm}<R_{\mathrm{a}}<50 \mathrm{~nm}$, and $R_{\mathrm{a}}>50 \mathrm{~nm}$. Though the fatigue lives for all three groups varied strongly a clear trend is discernable (Figure 8). Samples with average surface roughness lower than $30 \mathrm{~nm}$ exhibited the longest fatigue life, samples with a surface roughness between $30 \mathrm{~nm}$ and $50 \mathrm{~nm}$ exhibited a median fatigue life, and samples with surface roughness larger than $50 \mathrm{~nm}$ had the shortest average fatigue life. Samples that were mechanically polished with fine diamond slurry had the longest fatigue life and the lowest average surface roughness. For a summary of surface preparation, average surface roughness and fatigue life see Table 3.

\section{Discussion}

In this study, single crystal Ni-Mn-Ga samples were allowed to expand and contract up to $6 \%$ by careful design of the sample holder so that the ends of the sample were not constrained 
during magnetic field cycling. Using no constraint resulted in lower fatigue life for these samples compared to results reported in previous studies where strain was limited below 2\% [14]. Cracks preferentially propagated along macroscopic $<111>$ planes. The twin microstructure developed a complex pattern near fracture surfaces and areas of high stress concentration. High stress concentration prevents twin boundary movement resulting in a pileup of dislocations eventually forming cracks.

Using sulfuric acid as the electrolyte for electropolishing Ni-Mn-Ga, Chmielus et al. [26] found a reduction of twinning stress and lower surface roughness. When defects such as scratches are below $10 \mathrm{~nm}$, the average switching field (and hence twinning stress) is lowered and twin nucleation occurs more easily [27]. When surface defects are much larger there is a tendency for dislocations to become pinned and interact with other passing dislocations [18, 26]. Polishing with coarse slurry leads to a rougher surface and a stressed surface layer which allows for increased number of crack initiation points where dislocations will pile up and induce high stress concentration points. Higher surface stresses correlate with higher surface roughness and shorter fatigue life.

The crystals exhibiting longest fatigue life in studies performed by Aaltio et. al were linearly actuated $2 \times 10^{9}$ times with a controlled strain of $2 \%$ at a maximum stress of $2 \mathrm{MPa}$ [14]. By controlling and limiting the strain, only the twins with the highest mobility are active. In rotary cycling with permanent magnets, the sample is free to expand to the maximum possible strain (up to $6 \%$ for $10 \mathrm{M}$ martensite) depending on the applied field and condition of the sample. Higher strain results in twin boundaries moving longer distances and exposes twinning dislocations to more of the surface defects. This increases the probability of dislocations piling up at defects and increases stress concentrations. Eventually cracks nucleate, propagate and cause fracture [17].

The maximum magnetostress occurs in twins that withstand reorient due to barriers to twin boundary motion. In the present study, maximum magnetostress ranged from 3-4 $\mathrm{MPa}$ based on models from L'Vov and Chernenko[28-30]. The stress is lower in strain controlled linearly actuated samples because the sample is not fully elongated which reduces the interaction twinning dislocations with obstacles. Particularly, stresses did not exceed $2 \mathrm{MPa}$. Thus, the samples of this study experienced cyclical magnetostresses nearly twice as high as samples in the strain controlled experiments of Aaltio et al. [14].

High cycles on the order of $10^{6}$ were achieved by Chmielus et. al in experiments with rotating magnetic field reaching strains of approximately 1\% [15]. The samples in those experiments were constrained on both ends due to fixing the sample with glue to the sample holder. The resulting clamping forces limited the maximum strain. Chmielus et al. tested untrained samples with a finely twinned self-accommodated structure and effectively trained samples with nearly single twin variant microstructure. The samples with a fine twin microstructure showed the longest fatigue life as opposed to magneto-mechanical and thermomechanically trained samples, which fail in as little as 1,000 cycles [17]. Near the constraints, the microstructure remained locked in place, limiting the maximum strain. Applying those 
findings to the present results indicates that exposing the samples to a large magnetic field during measurement of magnetic properties prior to fatigue testing has effectively trained the samples which contributes to the short fatigue life.

In notched samples, the stress concentration is higher for the horizontal notches parallel to the [100] direction than for the notches along [110]. For the majority of samples, the cracks propagated from [100] notches and not from the [110] notches. The [100] notches are loaded in mode I (i.e. loading under tensile stress) while the [110] notches are loaded in a mixed mode containing mode I and mode II components (i.e. loading under shear stress and tensile stress). Therefore, the shear stress on the fracture plane is highest for [100] notches. A refined twin structure near defects indicates that these areas experience high stress concentrations (Figure 9). Cracks nucleated at the notch tip where the stress concentrations are highest.

The twin structure becomes complex near fracture surfaces and small angle grain boundaries (SAGBs). Near areas of high stress concentration, the microstructure evolves into a refined structure in order to accommodate inhomogeneous strains and to relax the stress. This refined structure presents obstacles for the motion of the mobile twin boundaries. The refined twin structure prevents reorientation due to high concentrations of twinning dislocations, resulting in higher cyclical effective stress in immobile twins and aiding crack formation.

The SAGBs do not completely hinder twin boundary motion. As a twin crosses an SAGB, it may break up into many smaller twins (Figure 10a). The higher number of twin boundaries located on the SAGB lead to stress concentrations due to an increased number of twinning dislocations. The twinning plane can also kink across a SAGB (Figure 10b), which involves distortions and, thus, stress concentrations. These areas of high stress concentration lead to crack nucleation and growth.

The fatigue life in samples magnetomechanically cycled in the rotary testing instrument experienced short fatigue life due to a number of factors. Some of the samples contained a small number of SAGBs. Under cyclic loading, twinning dislocations accumulated at SAGBs leading to stress concentrations. High surface roughness due to mechanical polishing marks resulted in high concentrations of stress, inducing a complex twin structure and pile-ups of twinning dislocations. The high concentration of dislocations increased the probability for crack nucleation and propagation. The fatigue data indicate that high surface roughness leads to shorter fatigue life, however, there is a large range of the fatigue life between samples within each arbitrary surface roughness group leading to higher error and overlap between the groupings. More data is required to substantiate these conclusions.

Long fatigue life requires high crystal quality expressed in low density of crystal defects such as SAGBs, inclusions, preexisting cracks, and surface defects. It is important to prepare samples with the highest quality surface treatment available to reduce high stress concentration areas [31]. Finally, by controlling strain through the use of constraints or localized MFIS, samples can be expected to exhibit longer fatigue live. Our findings correlate with similar findings obtained for thermal shape memory alloys such as NiTi [32, 33]. 


\section{Summary and Conclusion}

A testing device was developed to study the fatigue life and fracture mechanics of NiMn-Ga single crystals by exposing many samples to a cyclic magnetic field. The sample holder was designed to hold up to 60 samples with low constraint so that $6 \%$ MFIS was achievable for each magnetic field cycle. Thirty four samples were prepared with different surface treatments to determine the effect of stressed surfaces with heightened roughness on fatigue life in a rotating magnetic field. Some samples were notched to study the effect of stress concentration on crack nucleation.

As demonstrated in previous studies, MSMAs can sustain very large numbers of loading cycles. This study shows that stress concentrations associated with polishing and surface roughness, notches, SAGBs, and other defects substantially reduces fatigue life. Such defects represent obstacles to twin boundary motion. The interplay of moving twin boundaries with these defects leads to the accumulation of twinning dislocations which increase the local stresses and eventually cause crack nucleation. Cracks propagate preferentially along planes of high shear stress. The macroscopic fracture plans are typically close to $\{111\}$. To achieve a long fatigue life, Ni-Mn-Ga crystals must be free of defects such as notches, small angle grain boundaries, and other sources of stress concentration.

\section{Acknowledgments}

The authors thank Eric Rhoads for assistance with single crystal growth, Phil Boysen for machining advice, and Sam Barker for assistance with machining. We acknowledge financial support of the US Department of Energy's Office of Basic Energy Sciences under Contract DEFG-02-07ER46396. 


\section{References}

[1] K. Ullakko, J.K. Huang, C. Kantner, R.C. O'Handley, V.V. Kokorin, Applied Physics Letters 69 (1996) 1966.

[2] P. Müllner, K. Ullakko, Physica Status Solidi (B) 208 (1998) R1-R2.

[3] P. Mullner, V.A. Chernenko, G. Kostorz, Journal of Applied Physics 95 (2004) 1531.

[4] A. Sozinov, N. Lanska, A. Soroka, W. Zou, Applied Physics Letters 102 (2013) 021902.

[5] M.A. Marioni, R.C. O’Handley, S.M. Allen, Applied Physics Letters 83 (2003) 3966-3968.

[6] A.R. Smith, J. Tellinen, K. Ullakko, Acta Materialia 80 (2014) 373-379.

[7] K. Ullakko, L. Wendell, A. Smith, P. Müllner, G. Hampikian, Smart Materials and Structures 21 (2012) 115020.

[8] A. Smith, A. Saren, J. Järvinen, K. Ullakko, Microfluidics and Nanofluidics (2015) 1-9.

[9] N.M. Bruno, C. Ciocanel, H.P. Feigenbaum, A. Waldauer, Smart Materials and Structures 21 (2012) 094018.

[10] X. Zhou, W. Li, H.P. Kunkel, G. Williams, Journal of Physics: Condensed Matter 16 (2004) L39.

[11] C.S. Watson, C. Hollar, K. Anderson, W.B. Knowlton, P. Müllner, Advanced Functional Materials 23 (2013) 3995-4001.

[12] P. Müllner, V.A. Chernenko, G. Kostorz, Materials Science and Engineering: A 387-389 (2004) 965-968.

[13] K. Ullakko, L. Straka, A. Sozinov, N. Lanska, A. Soroka, I. Aaltio, S.-P. Hannula, F. Tuomisto, Structural Changes in Ni-Mn-Ga due to Fatigue of over 2 billion cycles, International Conference of Ferromagnetic Shape Memory Alloys ICFSMA, Bilbao, Spain, 2009.

[14] I. Aaltio, A. Soroka, Y. Ge, O. Söderberg, S.-P. Hannula, Smart Materials and Structures 19 (2010) 075014.

[15] M. Chmielus, V.A. Chernenko, W.B. Knowlton, G. Kostorz, P. Müllner, The European Physical Journal Special Topics 158 (2008) 79-85.

[16] L. Straka, H. Hänninen, A. Soroka, Y. Ezer, Enhanced Fatigue Behavior of Ni-Mn-Ga Magnetic Shape Memory Alloys with Tailor-made Fine Twin Microstructure, International Conference on New Actuators, Bremen, Germany, 2012, p. 675.

[17] P. Müllner, V.A. Chernenko, D. Mukherji, G. Kostorz, MRS Proceedings 785 (2003) D12-12.

[18] M. Chmielus, P. Müllner, Materials Science Forum 177-201 (2011) 177-201.

[19] M. Chmielus, I. Glavatskyy, J.-U. Hoffmann, V.A. Chernenko, R. Schneider, P. Müllner, Scripta Materialia 64 (2011) 888-891.

[20] D. Kellis, A. Smith, K. Ullakko, P. Müllner, Journal of Crystal Growth 359 (2012) 64-68.

[21] O. Heczko, K. Ullakko, IEEE Transactions on Magnetics 37 (2001) 2672-2674.

[22] O. Heczko, L. Straka, V. Novak, S. Fähler, Journal of Applied Physics 107 (2010) 09 A914.

[23] K. Rolfs, M. Chmielus, J.M. Guldbakke, R.C. Wimpory, A. Raatz, W. Petry, P. Müllner, R. Schneider, Advanced Engineering Materials 14 (2012) 614-635.

[24] R.W. Hertzberg, R.P. Vinci, J.L. Hertzberg, Deformation and Fracture Mechanics of Engineering Materials, John Wiley \& Sons, Inc., MA, 2013, p. 281-282.

[25] L. Straka, O. Heczko, H. Seiner, N. Lanska, J. Drahokoupil, A. Soroka, S. Fähler, H. Hänninen, A. Sozinov, Acta Materialia 59 (2011) 7450-7463.

[26] M. Chmielus, K. Rolfs, R. Wimpory, W. Reimers, P. Müllner, R. Schneider, Acta Materialia 58 (2010) 3952-3962.

[27] I. Aaltio, X.W. Liu, M. Valden, K. Lahtonen, O. Söderberg, Y. Ge, S.P. Hannula, Journal of Alloys and Compounds 577 (2013) S367-S371. 
[28] V.A. L'vov, S.P. Zagorodnyuk, V.A. Chernenko, The European Physical Journal B 27 (2002) 55-62.

[29] V. Chernenko, V. L'vov, E. Cesari, P. McCormick, Materials Transactions 8 (2000) 928-932.

[30] V.A. Chernenko, V.A. L'vov, P. Müllner, G. Kostorz, T. Takagi, Physical Review B 69 (2004) 134410.

[31] M. Rahim, J. Frenzel, M. Frotscher, J. Pfetzing-Micklich, R. Steegmüller, M. Wohlschlögel, H. Mughrabi, G. Eggeler, Acta Materialia 61 (2013) 3667-3686.

[32] G.S.P. Cheung, B.W. Darvell, International Endodontic Journal 40 (2007) 612-618.

[33] S.W. Robertson, A.R. Pelton, R.O. Ritchie, International Materials Reviews 57 (2012) 1-36.

\section{Table headers}

Table 1 Composition range (atomic-\%) of the 10M portion of single crystals used in fatigue tests and compression tests. The first value in each column corresponds to the composition nearest to the seed end of the crystal and the second value corresponds to the $10 \mathrm{M}$ portion farthest from the seed. Crystals 1-5 were used in fatigue tests and Crystal 6 was used in compression testing.

Table 2 The effective twinning stress prior to fatigue testing for groups of samples with a specified surface preparation. Twinning stress is typically higher when the magnetic field is applied parallel to the sample face due to the geometry of the sample.

Table 3 Range in fatigue life and surface roughness for samples prepared with varying surface preparation. Samples with the lowest surface roughness were mechanically polished with the finest diamond slurry. Notched samples from group Crystal 5 were omitted from the cycle to failure analysis. 


\section{Figure Captions}

Figure 1: a) Rotary fatigue testing instrument consisting of a rotating sleeve and a stationary sample holder. The rotating sleeve contains two rare earth bar magnets and a cylindrical magnet magnetized across the diameter. The sample holder slides into the rotating sleeve and retains samples in a stationary position during testing such that the [100] direction of the single crystal is parallel to the axis of rotation and the [001] axis is perpendicular to the axis of rotation. b) The magnetic field parallel to the sample face (blue dots) and perpendicular to the sample face (black dots) as a function of spindle head rotation. The red lines indicate the typical switching field required to reorient twin boundaries.

Figure 2: XRD and EDS diagram for a $\mathrm{Ni}_{51.2} \mathrm{Mn}_{26.6} \mathrm{Ga}_{22.2}$ (at-\%, nominal concentration) single crystal used for fatigue tests. Data points represent crystal composition and shaded areas represent crystal structure. The face of the $(001)_{S}$ plane at crystal length $0 \mathrm{~mm}$ is the face that was exposed from cutting the seed off of the crystal. The data was obtained along the length of the crystal corresponding to the image of the crystal shown below the diagram. The letters in the bottom left corner of the diagram correspond to the locations of samples that were cut from the crystal.

Figure 3: Magnetization curves as a function of the effective magnetic field for a sample prior to (a,b) and after (c,d) cycling to 810,000 cycles; the curves were measured with the field parallel $(\mathrm{a}, \mathrm{c})$ and perpendicular $(\mathrm{b}, \mathrm{d})$ to the sample face. After the sample has been cycled many times the discontinuity in the magnetization curve was largely reduced indicating reduced twinning activity.

Figure 4: Composite DIC optical micrographs of samples after rotary fatigue, Crystal 1-B (180,000 cycles) and Crystal 3-F (60,000 cycles). Three different types of cracks are present on the faces of the samples: 1) Type I cracks propagating in the $\langle 110\rangle_{\mathrm{S}}$ direction.

2) Type II cracks propagating in a macroscopic [100] direction. 3) Type III cracks that follow small angle grain boundaries.

Figure 5: SEM image of Crystal 1-E $\{111\}$ macroscopic fracture plane. Fracture plane is characterized by jagged striations indicating brittle fracture. The river markings (outlined with white arrows) on the fracture surface point towards the crack initiation point, the internal defect is circled by the dotted line.

Figure 6: DIC optical micrograph of Crystal 4-J after 10,000 revolutions. Cracks propagated in the $[\overline{\mathbf{1 1 0}}]$ and the $[\overline{\mathbf{1 1 0}}]$ directions away from the $[100]_{\mathrm{S}}$ notch tip. The fracture plane separates two active portions of the sample where many twins are present on the left side of the cracks, but only few twins are present on the right side. 
Figure 7: Optical micrograph using DIC of sample Crystal 1-E after 80,000 cycles. Twin microstructure farthest from the crack is composed of compound twins within thick type II twins. The compound twins are continuous and extend across the type II twin boundaries as indicated by the white dashed lines. Near the fracture surface the twins decompose into a mixture of type I and II twins. The $c$-axis of the unit cell is parallel to [001] $\mathrm{S}$ within the green twin and parallel to $[010]_{S}$ within the pink and orange twins.

Figure 8: Diagram representing the number of revolutions to failure, blue bars represent the average number of revolutions to fracture, the black error bars are the standard deviation of the average, and the range between minimum and maximum number of revolutions for each group is represented by the gold bars.

Figure 9: DIC image of sample Crystal 4-L after 12,000 cycles. The twin structure near the notch (black arrows) is refined containing Type I and II twins in a variety of orientations. The twin structure within the bulk material contains larger mobile twins.

Figure 10: DIC microscopy images of Crystal 4-A after 40,000 cycles. a) Twins decompose into multiple fine twins across the SAGB. b) Twin boundaries are kinked where they cross over SAGBs. SAGBs indicated by white dotted line. 
Fatigue life and fracture mechanics of unconstrained Ni-Mn-Ga single crystals in a rotating magnetic field

\section{T. Lawrence ${ }^{1}$, P. Lindquist ${ }^{1}$, K. Ullakko ${ }^{2}$, P. Müllner ${ }^{1}$}

${ }^{1}$ Department of Materials Science and Engineering, Boise State University, Boise, ID 83725, USA

${ }^{2}$ Lappeenranta University of Technology, LUT Chemtech, Savonlinna, Finland

Figures
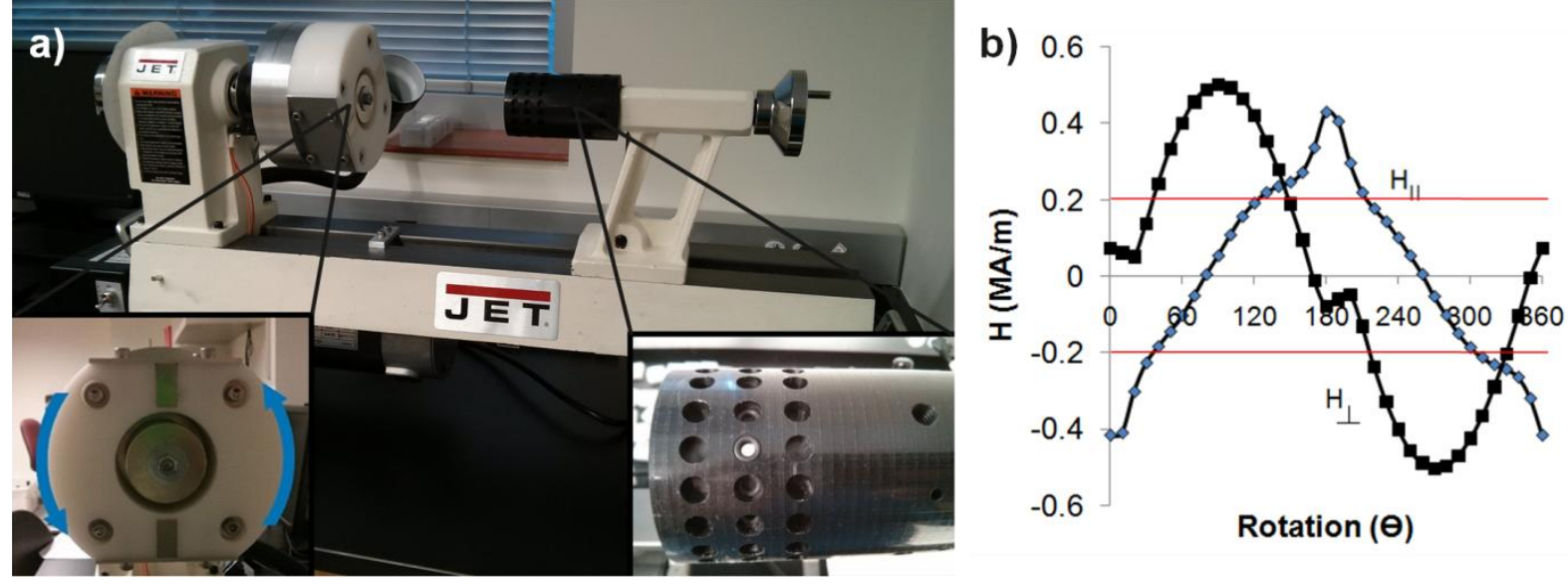

Figure 1 


\section{132}

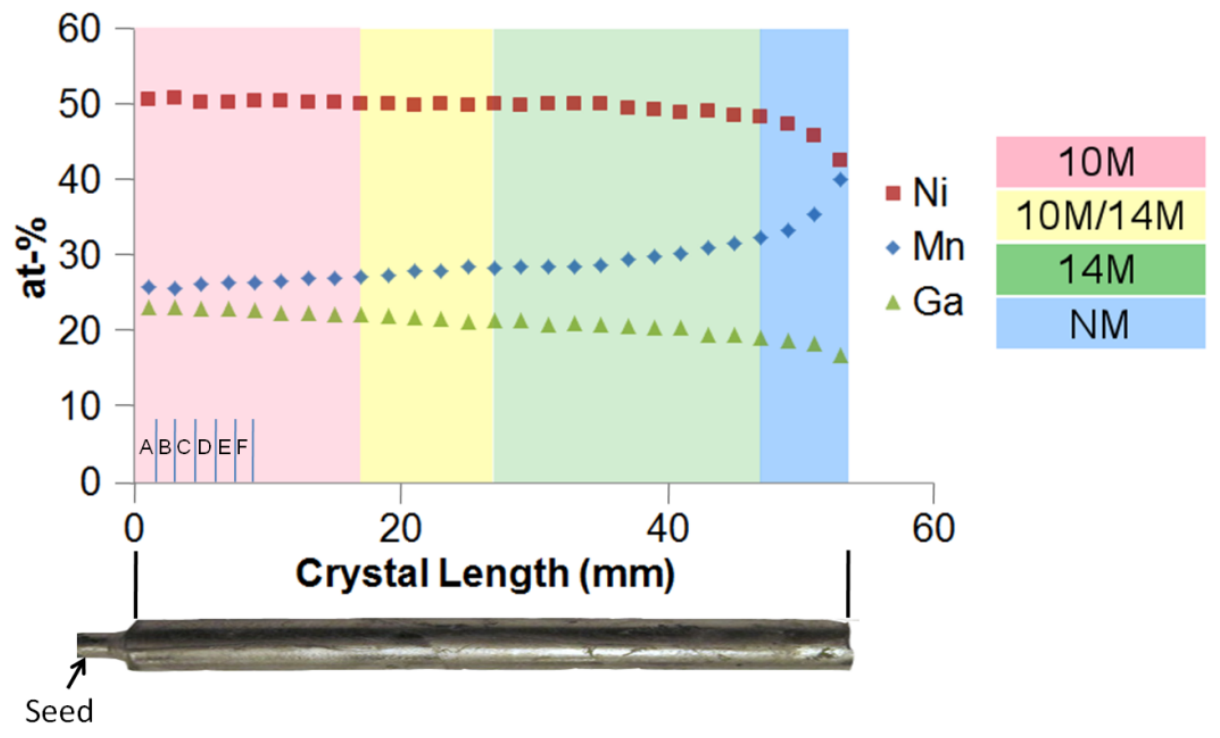

Figure 2 
a)

Field Parallel to Face Initial

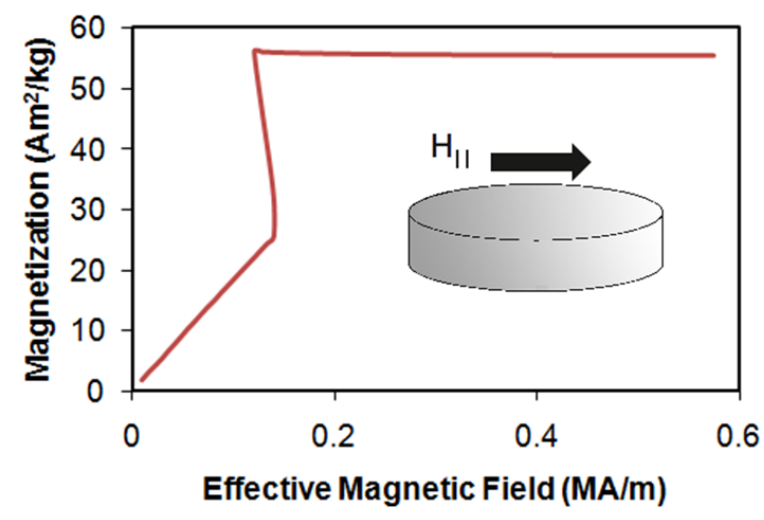

c)

Field Parallel to Face 810,000 Cycles

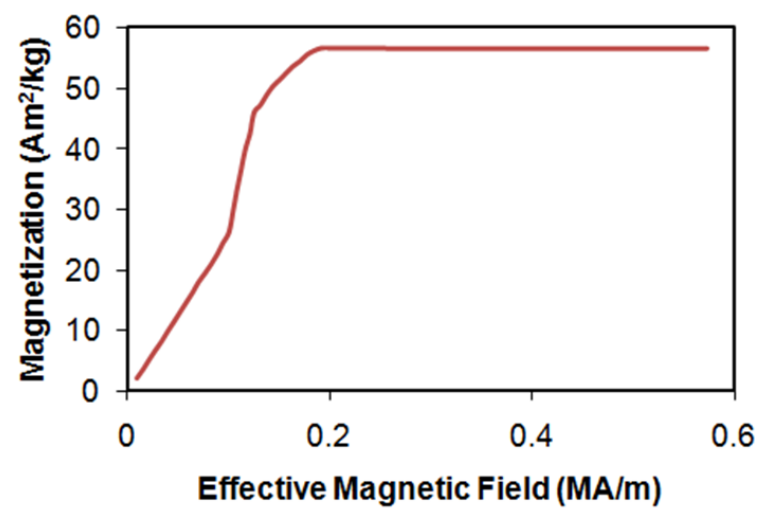

b) Field Perpendicular to Face Initial

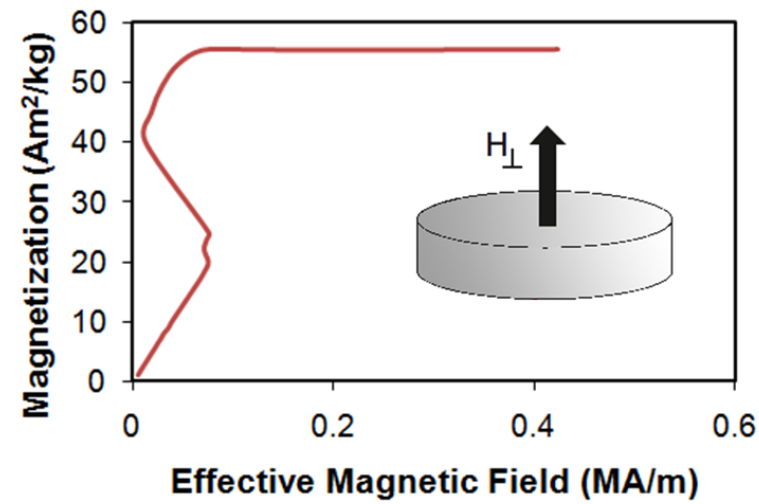

d) Field Perpendicular to Face $\mathbf{8 1 0 , 0 0 0}$ Cycles

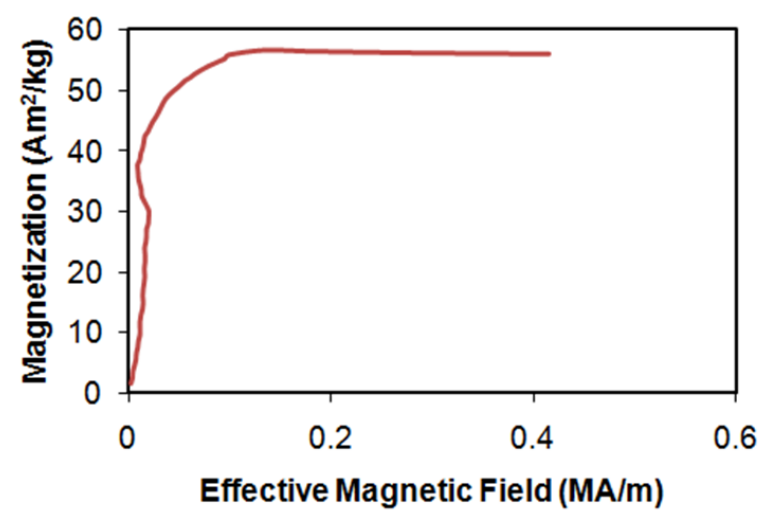

Figure 3 


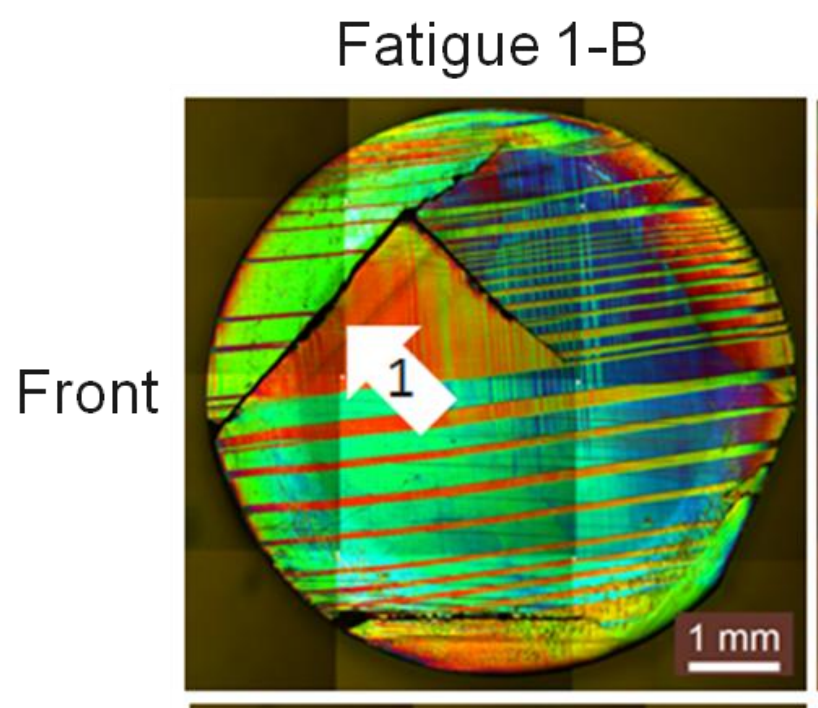

Fatigue 3-F
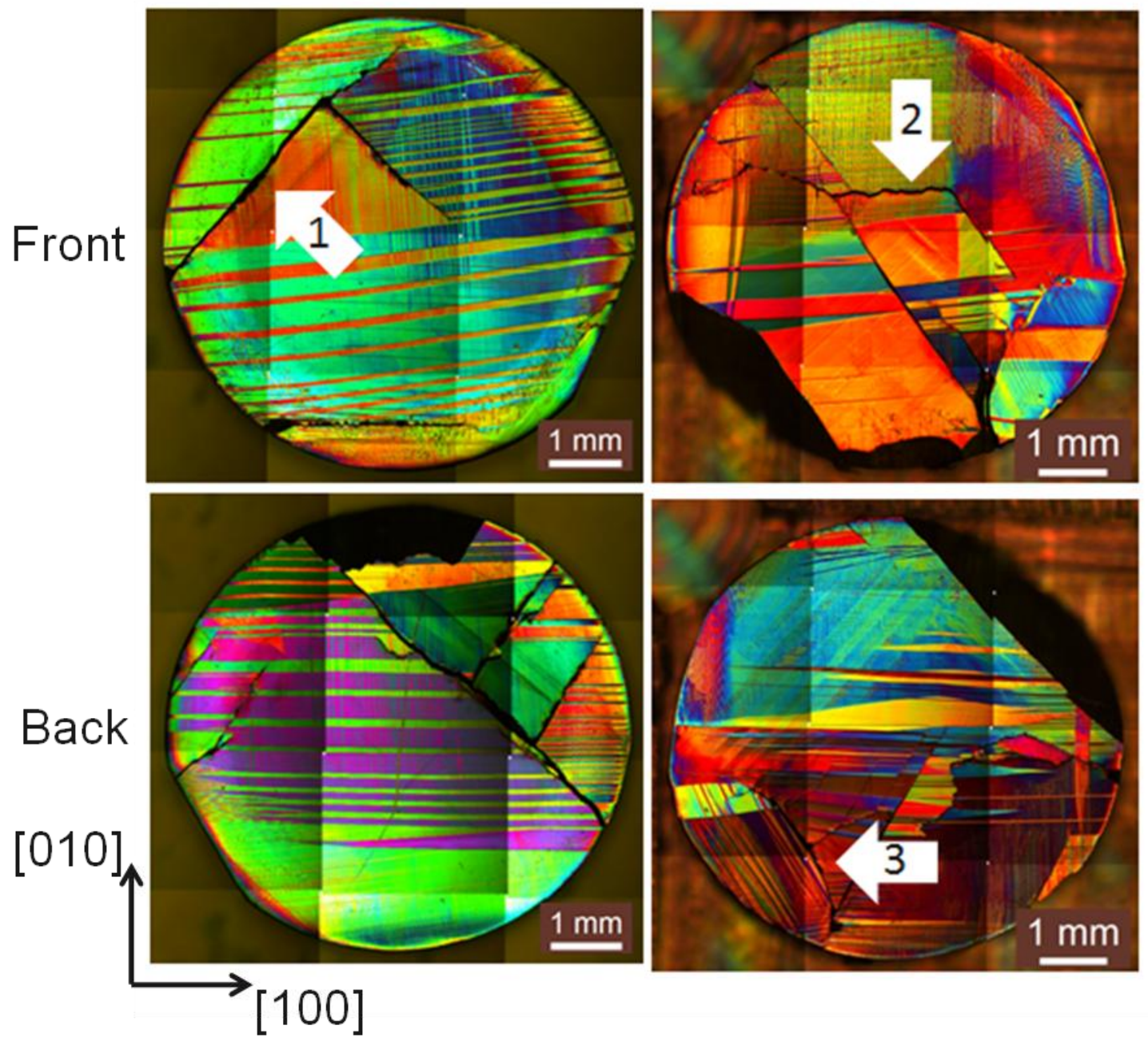

Figure 4 


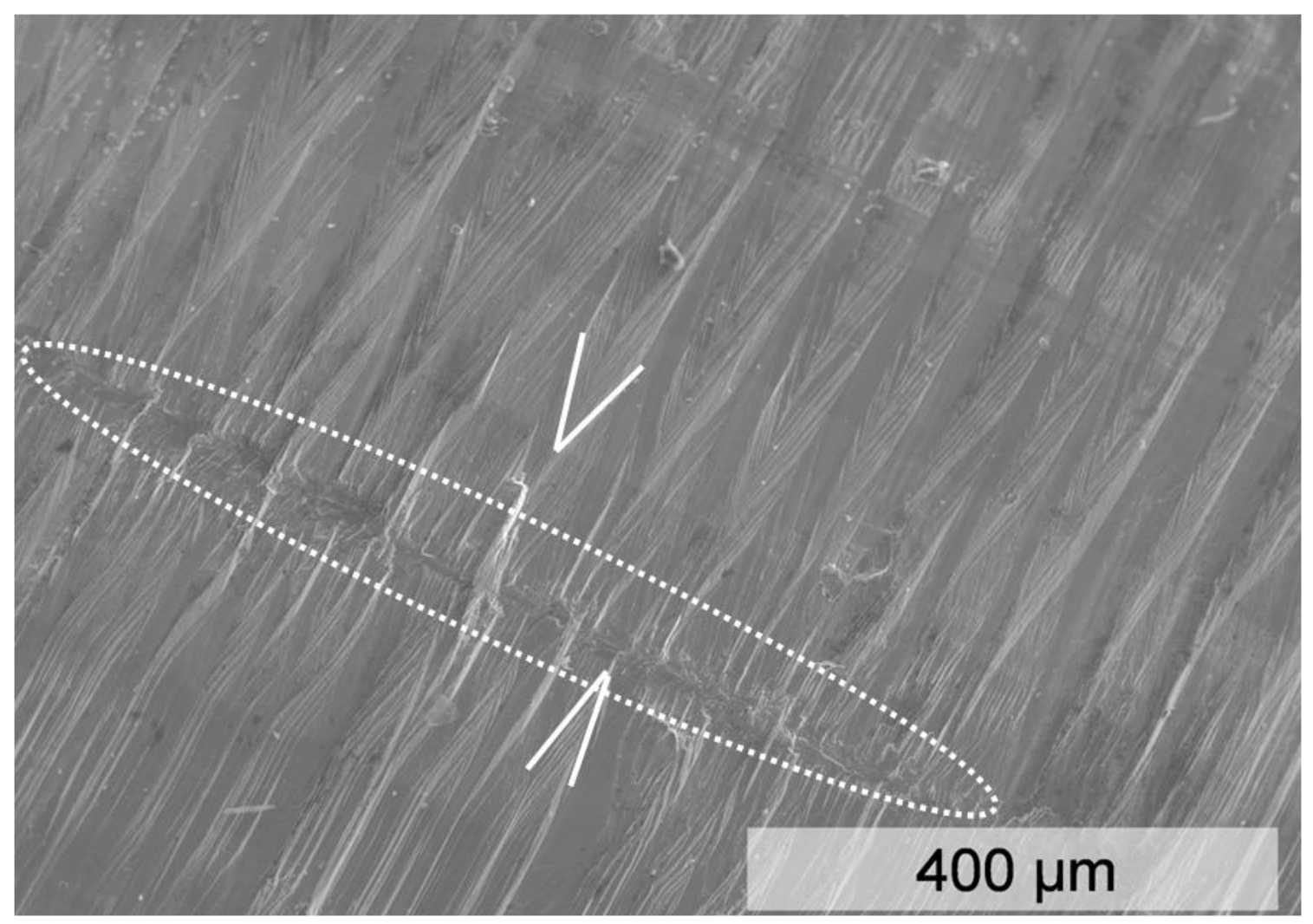

Figure 5 


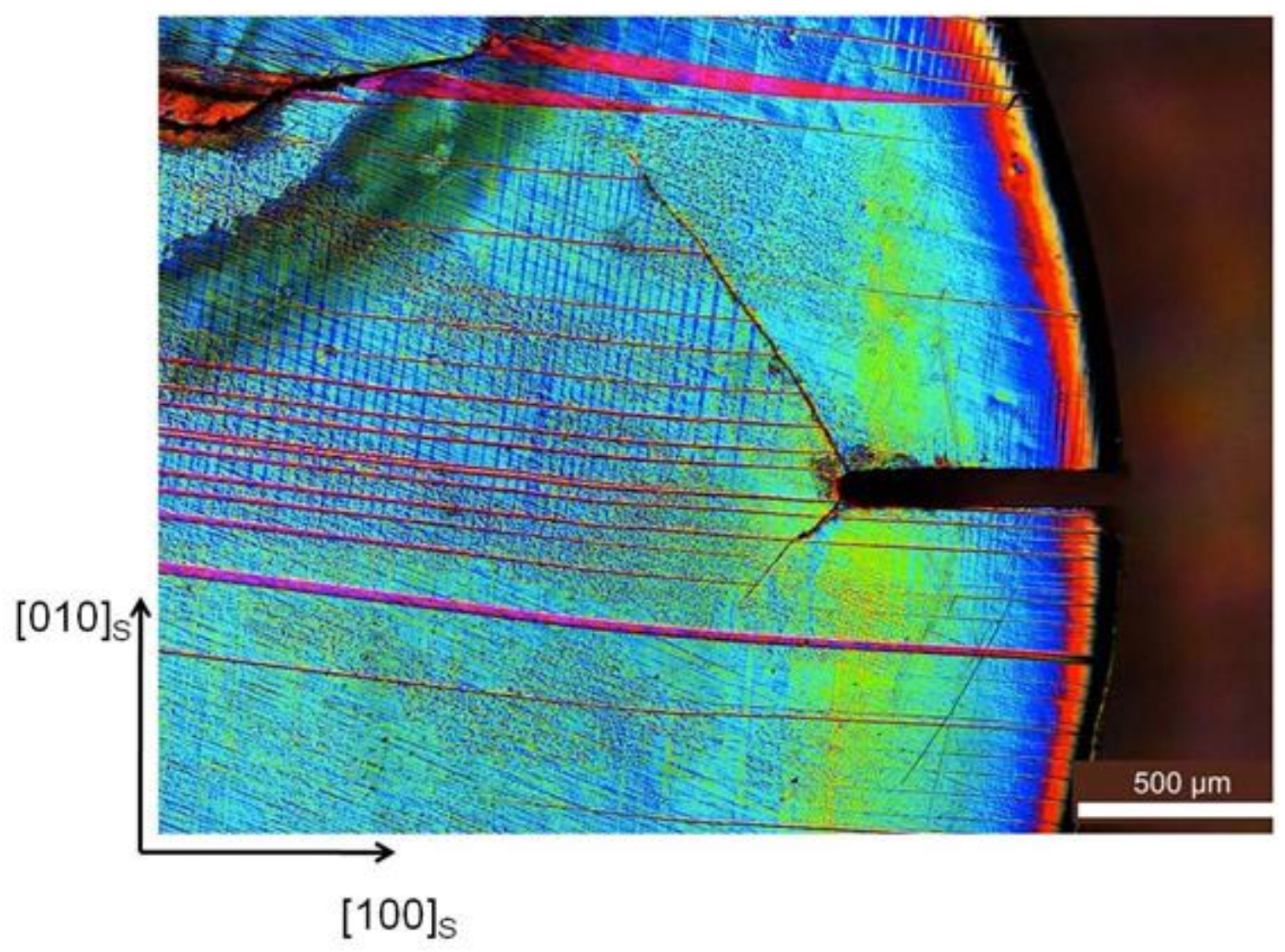

Figure 6 


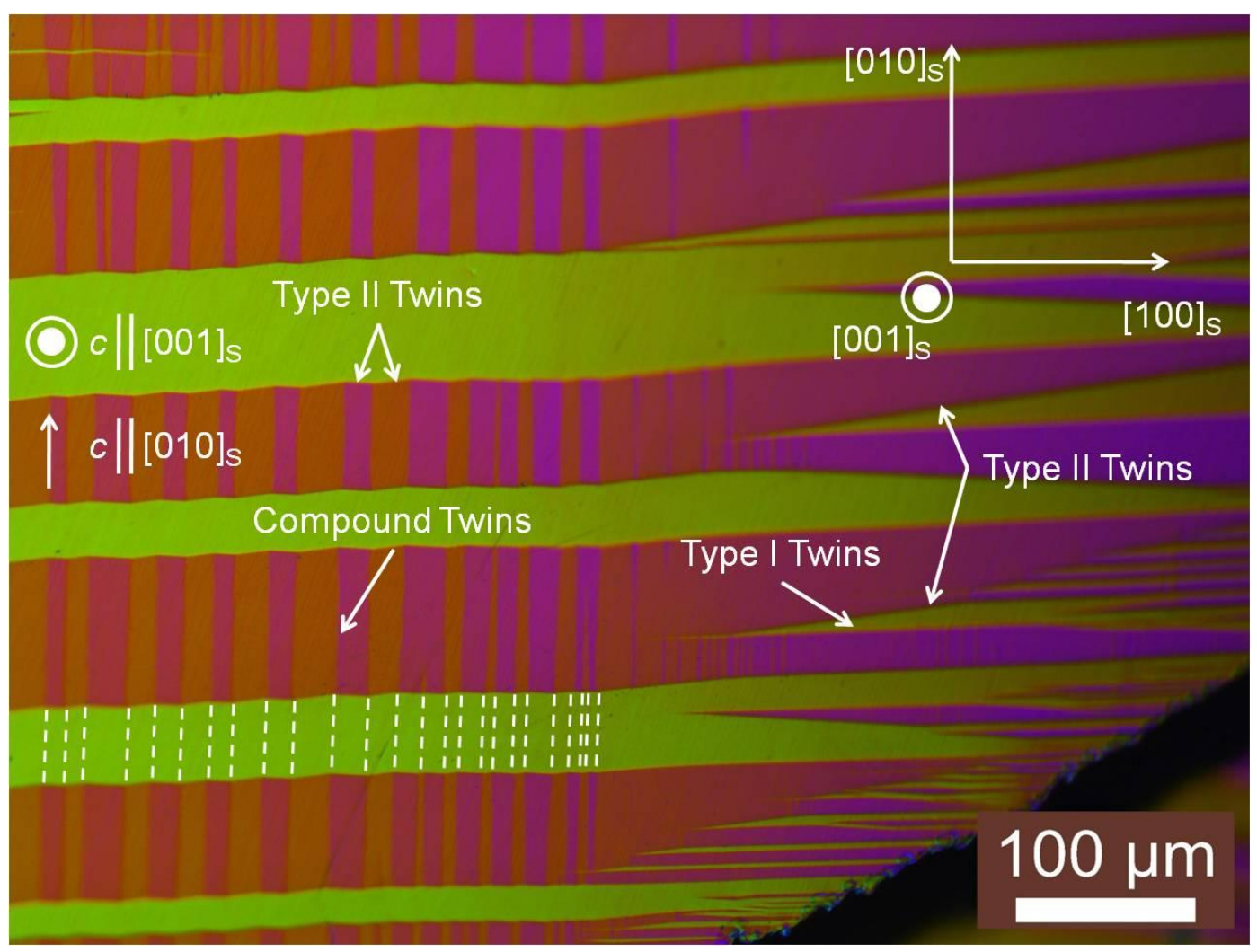

Figure 7 


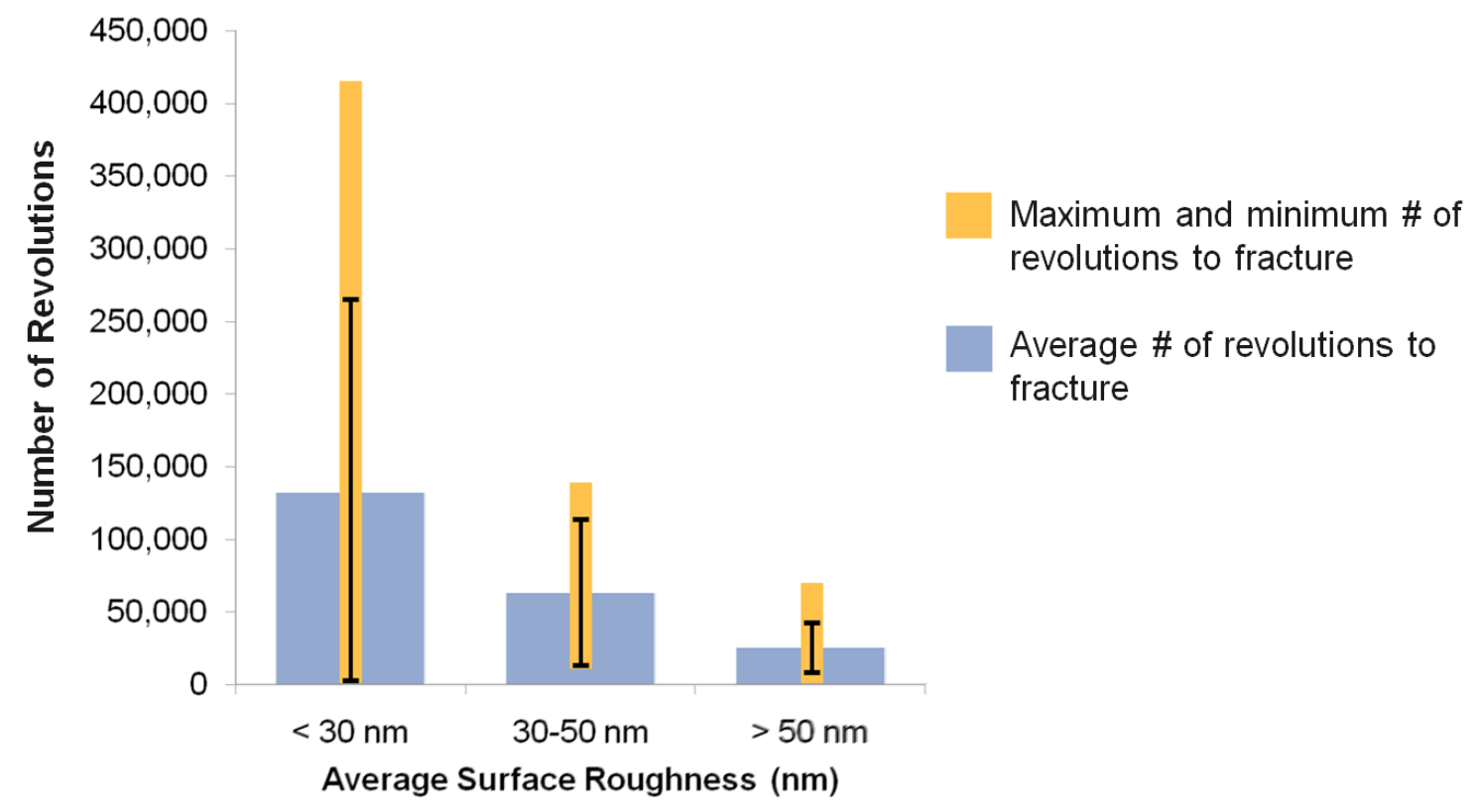

Figure 8 


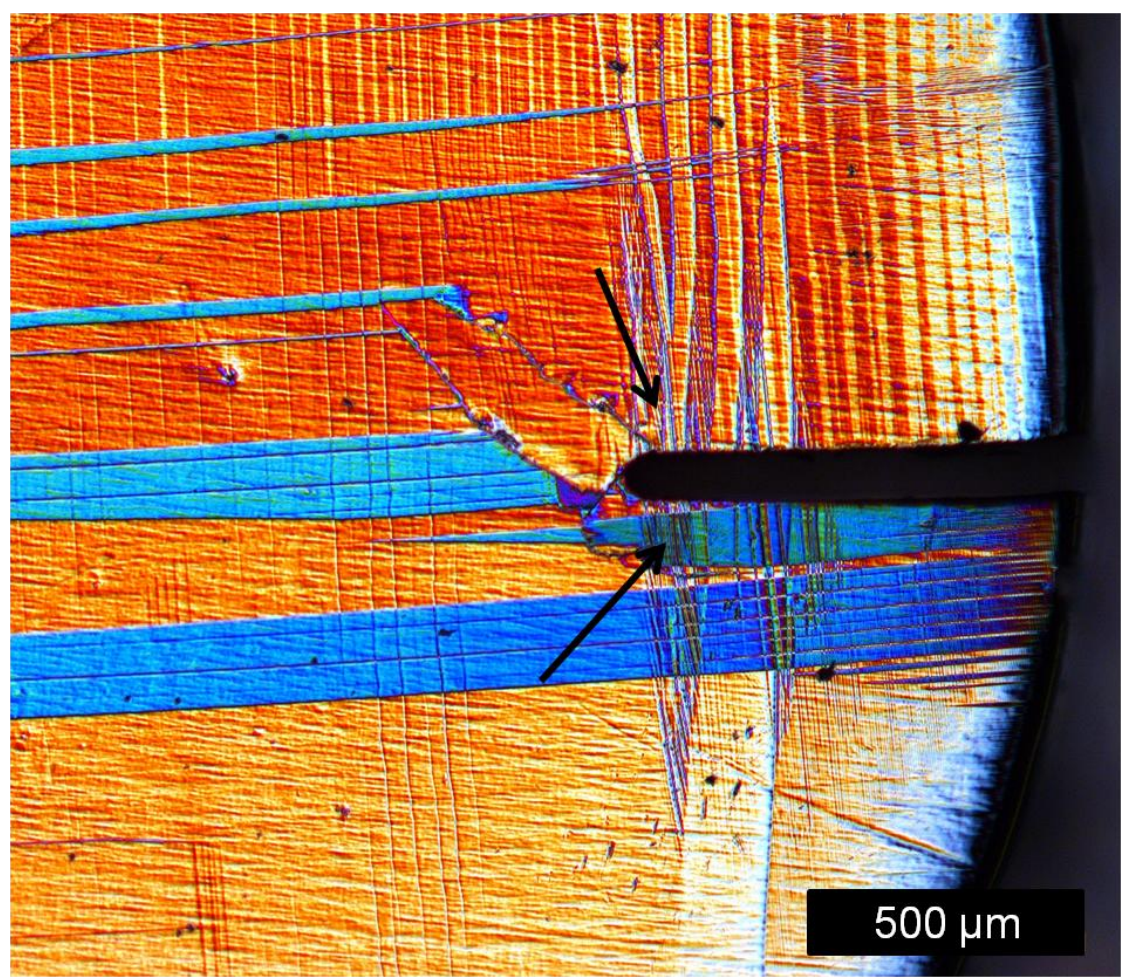

Figure 9 

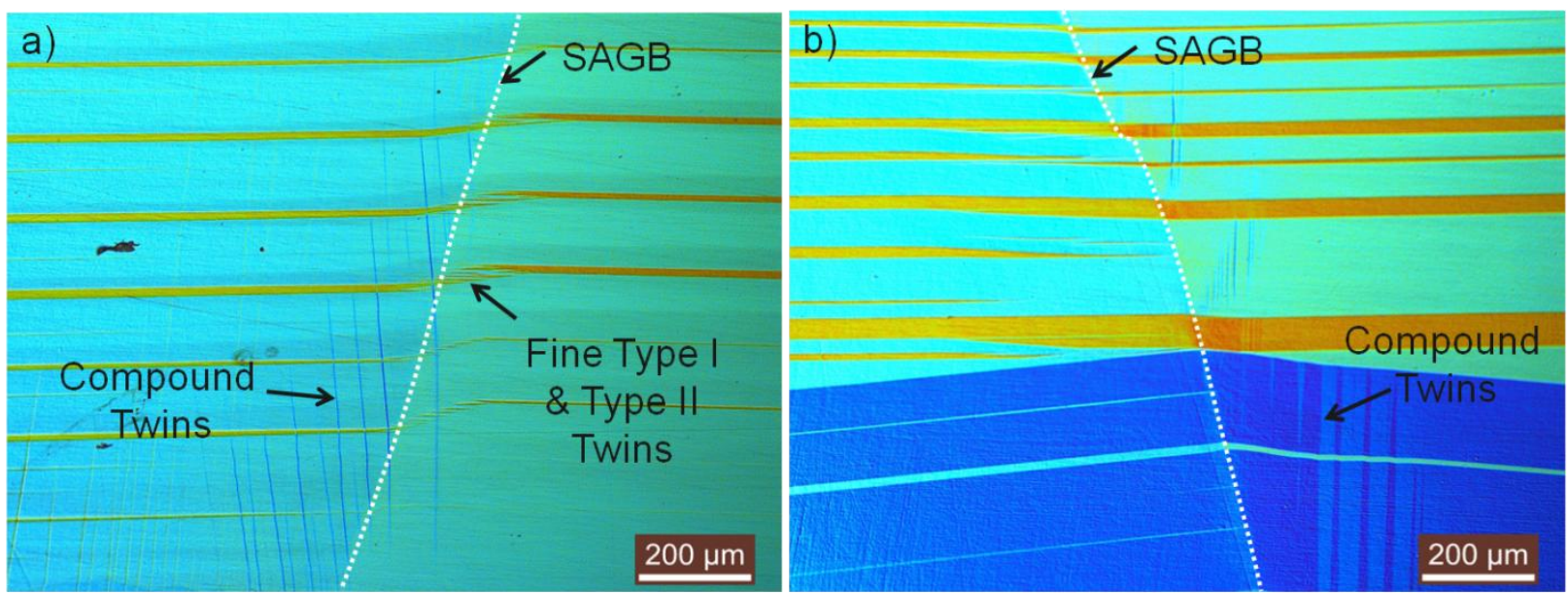

Figure 10 
Fatigue life and fracture mechanics of unconstrained Ni-Mn-Ga single crystals in a rotating magnetic field

\author{
T. Lawrence ${ }^{1}$, P. Lindquist ${ }^{1}$, K. Ullakko ${ }^{2}$, P. Müllner ${ }^{1}$ \\ ${ }^{1}$ Department of Materials Science and Engineering, Boise State University, Boise, ID 83725, \\ USA \\ ${ }^{2}$ Lappeenranta University of Technology, LUT Chemtech, Savonlinna, Finland
}

Table 1 Composition range (atomic-\%) of the 10M portion of single crystals used in fatigue tests and compression tests. The first value in each column corresponds to the composition nearest to the seed end of the crystal and the second value corresponds to the $10 \mathrm{M}$ portion farthest from the seed. Crystals 1-5 were used in fatigue tests and Crystal 6 was used in compression testing.

\begin{tabular}{|c|c|c|c|}
\hline Single Crystal & Ni (at-\%) & Mn (at-\%) & Ga (at-\%) \\
\hline Crystal 1 & $50.8-50.4 \pm 0.2$ & $25.9-27.2 \pm 0.2$ & $23.3-22.4 \pm 0.2$ \\
\hline Crystal 2 & $51.1-50.1 \pm 0.2$ & $24.8-25.6 \pm 0.2$ & $24.1-23.7 \pm 0.2$ \\
\hline Crystal 3 & $51.2-50.9 \pm 0.2$ & $24.8-25.4 \pm 0.2$ & $24.0-23.7 \pm 0.2$ \\
\hline Crystal 4 & $51.5-51.1 \pm 0.2$ & $25.0-26.6 \pm 0.2$ & $23.5-22.3 \pm 0.2$ \\
\hline Crystal 5 & $51.7-51.6 \pm 0.2$ & $25.5-26.5 \pm 0.2$ & $22.8-22.0 \pm 0.2$ \\
\hline Crystal 6 & $51.6-51.4 \pm 0.2$ & $25.8-27.2 \pm 0.2$ & $22.7-21.4 \pm 0.2$ \\
\hline
\end{tabular}


Table 2 The effective twinning stress prior to fatigue testing for groups of samples with a specified surface preparation. Twinning stress is typically higher when the magnetic field is applied parallel to the sample face due to the geometry of the sample.

\begin{tabular}{|c|c|c|c|}
\hline $\begin{array}{l}\text { Single } \\
\text { crystals }\end{array}$ & $\begin{array}{c}\text { Surface } \\
\text { preparation }\end{array}$ & $\begin{array}{c}\text { Effective } \\
\text { twinning stress } \\
\text { with magnetic } \\
\text { field parallel to } \\
\text { sample face } \\
(\mathrm{MPa})\end{array}$ & $\begin{array}{c}\text { Effective } \\
\text { twinning stress } \\
\text { with magnetic } \\
\text { field } \\
\text { perpendicular to } \\
\text { sample face } \\
(\mathrm{MPa})\end{array}$ \\
\hline Crystal 1 & $3 \mu \mathrm{m}$ & $0.4 \pm 0.2$ & $0.6 \pm 0.2$ \\
\hline Crystal 1 & $1 \mu \mathrm{m}$ & $0.20 \pm 0.07$ & $0.66 \pm 0.02$ \\
\hline Crystal 2 & $3 \mu \mathrm{m}$ & $0.3 \pm 0.1$ & $0.3 \pm 0.2$ \\
\hline Crystal 3 & $3 \mu \mathrm{m}$ & $0.3 \pm 0.2$ & $0.7 \pm 0.4$ \\
\hline Crystal 4 & $3 \mu \mathrm{m}$ & $0.30 \pm 0.09$ & $0.5 \pm 0.2$ \\
\hline Crystal 4 & $0.04 \mu \mathrm{m}$ & $0.27 \pm 0.07$ & $0.50 \pm 0.07$ \\
\hline Crystal 5 & $3 \mu \mathrm{m}$ & $0.3 \pm 0.1$ & $0.24 \pm 0.06$ \\
\hline
\end{tabular}


Table 3 Range in fatigue life and surface roughness for samples prepared with varying surface preparation. Samples with the lowest surface roughness were mechanically polished with the finest diamond slurry. Notched samples from group Crystal 5 were omitted from the cycle to failure analysis.

\begin{tabular}{|c|c|c|c|}
\hline Single Crystal & $\begin{array}{c}\text { Sample } \\
\text { Preparation }\end{array}$ & $\begin{array}{c}\text { Ra, Average } \\
\text { Surface } \\
\text { Roughness (nm) }\end{array}$ & $\begin{array}{c}\text { Average Number of } \\
\text { Cycles to Fracture }\end{array}$ \\
\hline Crystal 1 & $3 \mu \mathrm{m}$ & $22 \pm 5$ & $66,000 \pm 65,483$ \\
\hline Crystal 1 & $1 \mu \mathrm{m}$ & $14 \pm 7$ & $53,333 \pm 15,275$ \\
\hline Crystal 2 & $3 \mu \mathrm{m}$ & $35 \pm 16$ & $30,000 \pm 20,976$ \\
\hline Crystal 3 & $3 \mu \mathrm{m}$ & $29 \pm 6$ & $189,333 \pm 195,308$ \\
\hline Crystal 4 & $3 \mu \mathrm{m}$ & $77 \pm 44$ & $97,857 \pm 120,046$ \\
\hline Crystal 4 & $0.04 \mu \mathrm{m}$ & $16 \pm 6$ & $153,000 \pm 149,231$ \\
\hline Crystal 5 & $3 \mu \mathrm{m}$ & $19 \pm 9$ & N/A \\
\hline & & & \\
\hline
\end{tabular}

